\title{
Performance of a $4 \pi$ Ge spectrometer for neutron capture cross section measurements
}

\author{
M. Oshima ${ }^{1, a}$, J. Hori ${ }^{2}$, H. Harada ${ }^{1}$, K. Furutaka ${ }^{1}$, M. Koizumi ${ }^{1}$, F. Kitatani ${ }^{1}$, Y. Toh ${ }^{1}$, A. Kimura ${ }^{1}$, S. Nakamura ${ }^{1}$, \\ M. Igashira ${ }^{3}$, M. Mizumoto ${ }^{3}$, T. Ohsaki ${ }^{3}$, T. Katabuchi ${ }^{3}$, J. Nishiyama ${ }^{3}$, and J. Goto ${ }^{4}$ \\ Japan Atomic Energy Agency, Tokai, Ibaraki 319-1195, Japan \\ 2 Nuclear Reactor Institute, Kyoto University, Kumatori, Sennan, Osaka 590-0494, Japan \\ 3 Research Laboratory for Nuclear Reactor, Tokyo Institute of Technology, O-okayama, Meguro, Tokyo 152-8550, Japan \\ ${ }^{4}$ Niigata University, Asahimachi-dori, Niigata, Niigata 590-0494, Japan
}

\begin{abstract}
Neutron capture cross sections of minor actinides and long-lived fission products are important for the R\&D of innovative nuclear reactors. However, those data are rather poor both in quality and in quantity at present. Thus, we have started projects for neutron cross section measurements. We have constructed an $4 \pi$ Ge spectrometer consisting of Ge crystals and BGO shields. The neutron cross section measurements have been done at KURRI electron linac and JAEA JRR-3 facilities, which will be extended at the high-intensity neutron source at J-PARC.
\end{abstract}

\section{Introduction}

Neutron capture cross sections of minor actinides (MA) and long-lived fission products (LLFP) are very important for the $\mathrm{R} \& \mathrm{D}$ of innovative nuclear reactors such as Fast Breeder Reactors or Accelerator Driven Systems. However, those data are rather poor both in quality and in quantity at present. Thus, we proposed a project entitled "Fundamental R\&D on Neutron Cross Sections for Innovative Reactors using Advanced Radiation Measurement Technology" by the Ministry of Education, Culture, Sports, Science and Technology in Japan and it was approved as one of "Innovative Nuclear Energy System Technology Development Projects" [1]. An essential part of this project is the development of advanced measurement technology which is demonstrated by sophisticated $\gamma$-ray detector arrays such as GAMMASPHERE [2,3] and EUROBALL [4]. Hence we have constructed a $4 \pi \mathrm{Ge}$ spectrometer consisting of HPGe crystals and BGO shields for the measurement of neutron capture cross sections.

In neutron capture cross section measurement there are several backgrounds such as $\gamma$-rays from impurities in the sample, scattered neutrons from the sample, neutron room background and so on. Although we use a sample purified as much as possible, a mixture of other nuclides in the order of a few percent is inevitable. Since the neutron capture cross section often varies from nuclide to nuclide as large as one or two orders of magnitude, this unavoidable mixture poses a certain limit on the accuracy of measurements. Therefore, the discrimination of nuclides is crucial to achieve the accuracy of a few percent in the measurement of neutron capture cross sections of MA and LLFP.

After the neutron capture reaction the excited nucleus emits plural $\gamma$-rays in cascade. Since the nucleus has a proper excited level structure, the $\gamma$-cascade itself is peculiar. Thus the best signature for the identification of neutron capture events is the single $\gamma$-rays emitted from each nucleus. For this purpose high-resolution Ge detectors are expected to play

\footnotetext{
${ }^{a}$ Presenting author, e-mail: oshima.masumi@jaea.go.jp
}

a crucial role in the high precision determination of neutron cross sections. Nevertheless, the use of Ge detectors is scarce so that we try to apply large Ge detector array to the neutron capture experiments. The conceptual design studies have been already made in refs. [5,6].

\section{Basic performance of $4 \pi$ Ge spectrometer}

We have constructed a $4 \pi$ Ge spectrometer for neutron capture cross section measurements, which comprises two EUROBALL cluster and four clover germanium detectors surrounded by BGO anti-Compton shields. The cluster detectors consist of seven hexagonal crystals and each has the efficiencies between $57 \%$ and $65 \%$ relative to a 3 inch $\phi \times$ 3 inch NaI scintillator. Also each clover detector has four crystals inside and each has the relative efficiencies between $23 \%$ and $27 \%$. The number of high resolution outputs from the $\mathrm{Ge}$ detectors is 42 including 12 outputs from segmented cluster module.

At first the spectrometer was set at the neutron beam facility in the research reactor, JRR-3, in Japan Atomic Energy Agency (JAEA). Figure 1 shows a whole view of the spectrometer. The cold neutron beam with typical $1.5 \mathrm{meV}$ comes

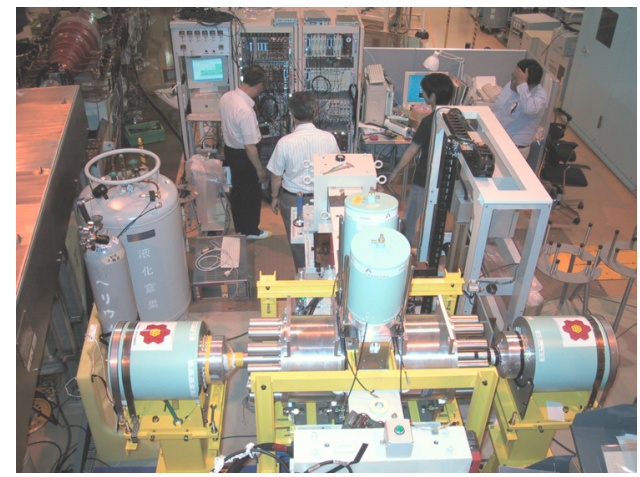

Fig. 1. The whole view of the $4 \pi$ Ge spectrometer. 


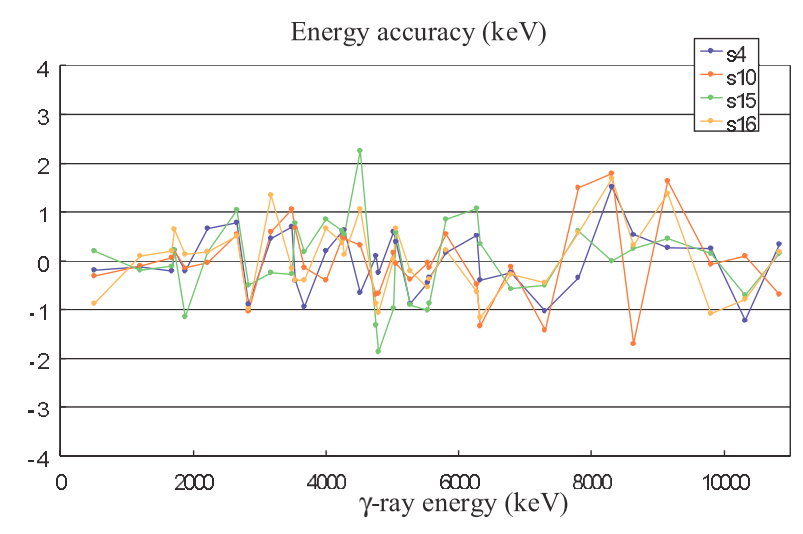

Fig. 2. Gamma-ray energy determination accuracy of typical four outputs from the $4 \pi$ Ge spectrometer.

from the bottom to the beam stop shown at the center of the photo. At both sides of the figure a liquid nitrogen dewar for Ge detectors and an automatic sample changer can be seen. Behind the beam stop there is a data acquisition system.

We have developed a new data acquisition system because we deal with high-resolution outputs from the Ge detectors in coincidence mode and those from BGO Compton suppressors in anti-coincidence mode; they need be processed at high counting rate (in the order of $100 \mathrm{kHz}$ ). Conventional data acquisition systems cannot handle such high rate and, in addition, require large space and high cost. Our new system utilizes the rapidly developing digital signal processing techniques and now in operation [7]. By applying the system to the $4 \pi$ Ge spectrometer we could achieve the energy resolution of $2.5 \mathrm{keV}$ FWHM at $1.33 \mathrm{meV}$ and $16.4 \mathrm{keV}$ FWHM at $10.83 \mathrm{meV}$. A detailed report is presented in ref. [8].

\subsection{Energy resolution}

The energy resolution is measured by using prompt $\gamma$-rays from the ${ }^{14} \mathrm{~N}($ thermal $\mathrm{n}, \gamma){ }^{15} \mathrm{~N}$ reaction. Typically the $2.8 \mathrm{keV}$ and $14 \mathrm{keV}$ FWHM has been derived at 1 and $10 \mathrm{meV}$, respectively. Including the ADC linearity we could derive the $\gamma$-ray determination accuracy as shown in figure 2. Considering a wide dynamic range, we could obtain a reasonable accuracy for the $\gamma$-ray energy determination.

\subsection{Detection efficiency}

The absolute detection efficiency for a single $\gamma$-ray depends much on the analysis procedure. The spectrometer consists of two EUROBALL cluster detectors and four clover detectors; the cluster detector includes one center crystal and six outer crystals; and the clover detector includes four crystals. Hence there are 30 outputs from individual crystals. The crystals are closely packed so that cross talk events cannot be neglected originating from the $\gamma$-ray events Compton-scattered in one crystal and absorbed in the other. Thus we evaluated the absolute efficiencies of the spectrometer in the following three cases: (a) all the signals are summed up event-by-event,
Efficiency of single gamma rays

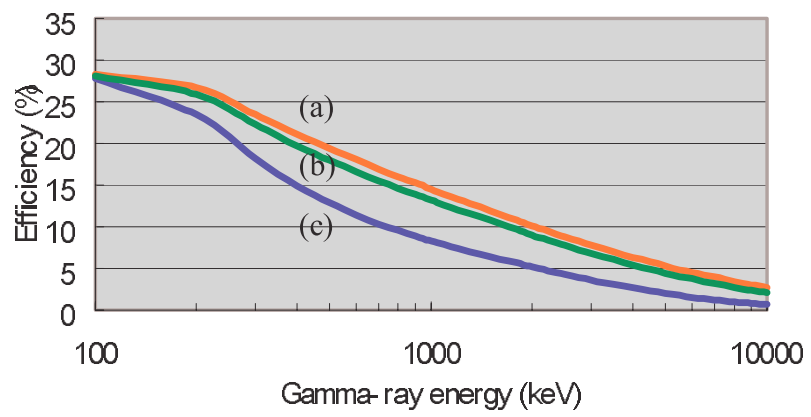

Fig. 3. Absolute efficiency of the $4 \pi$ Ge spectrometer for the three cases, (a) all the signals are summed up event-by-event, (b) the signals from each (cluster and claver) detector modules are summed up, and (c) all the signals are treated individually.

(b) the signals from each (cluster and clover) detector modules are summed up, and (c) all the signals are treated individually. The efficiency is calculated with the Monte Carlo simulation code GEANT [9] and shown in figure 3. The efficiency for (a) amounts to $15 \%$ at $1 \mathrm{meV}$. Thus the spectrometer has turned to be one of the most efficient high-resolution $\gamma$-ray detector arrays at present.

The major purpose to apply Ge detectors to neutron capture cross section measurements is nuclide discrimination. There are several ways to achieve this. The first one is to use total energy of the $\gamma$-cascade by which the product nucleus deexcites to its ground state. For this method $4 \pi$ calorimeters made of liquid or $\mathrm{BaF}_{2}$ scintillators have been developed. The most powerful calorimeter is the ones with detection efficiency larger than $90 \%[10,11]$. Their energy resolution ranges from $14 \%$ at $662 \mathrm{keV}$ to $6 \%$ at $6.13 \mathrm{meV}$. The use of our $4 \pi \mathrm{Ge}$ spectrometer as a calorimeter is, however, not straightforward, because of its small detection efficiency. As shown in figure 3, the detection efficiency for a single $\gamma$-ray amounts to $15 \%$ at $1 \mathrm{meV}$ and it is in the order of $0.1 \%$ for triple $\gamma$-rays. There are non negligible cross talk events and it difficult to completely distinguish between the cross talk and independent hit of the $\gamma$-rays. This brings about considerable uncertainty to the final cross section.

The second choice is to use the $4 \pi$ Ge spectrometer as an array of single detectors. The energy resolution is more than one hundred times better than the $\mathrm{BaF}_{2}$ or $\mathrm{C}_{6} \mathrm{D}_{6}$ scintillators so that by measuring single discrete $\gamma$-rays we can easily discriminate nuclides. Furthermore, by measuring ground-state transitions, one can determine the neutron capture reaction rate accurately. In order for the detection efficiency to be defined unambiguously, it is important that the detectors are well separated from the $\gamma$-ray source and the effects of cross talk and double hits of $\gamma$-rays are negligible. For this method the detection efficiency of case (c) in figure 3 can apply.

\subsection{Application to time-of-flight experiment}

After the basic performance test, the spectrometer has been moved to the neutron time-of-flight (TOF) facility of the 


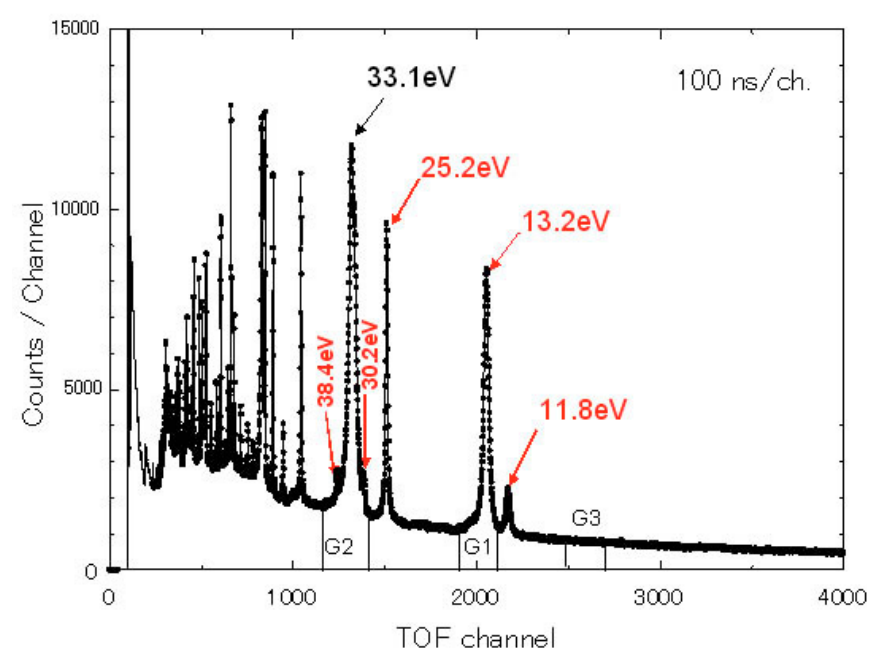

Fig. 4. A TOF spectrum obtained for ${ }^{\text {nat }} \mathrm{Pd}$ sample. Resonance peaks are observed for ${ }^{105} \mathrm{Pd}$ and ${ }^{108} \mathrm{Pd}$ nuclei.
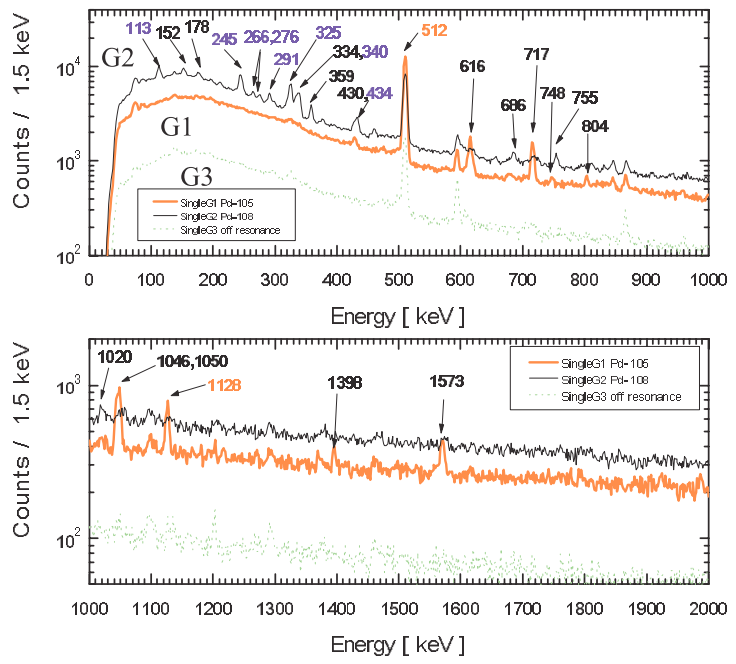

Fig. 5. $\gamma$-ray spectra gated for G1, G2 and G3 portion of TOF spectra in figure 4.

electron linac in Kyoto University Research Reactor Institute (KURRI). A pulsed electron beam of $30 \mathrm{MeV}$ is bombarded on the Ta target and produces fast neutrons. The repetition rate of the pulse is $100 \mathrm{~Hz}$ and the pulse width is $100 \mathrm{~ns}$. Typical beam intensity is $30.5 \mu \mathrm{A}$. The neutrons are cooled by a $\mathrm{H}_{2} \mathrm{O}$ moderator. At the $10 \mathrm{~m}$ off the target we put the sample and the prompt $\gamma$-rays are produced from the sample. The spectrometer is used to measure prompt $\gamma$-rays with high energy resolution.

For about $5 \mu \mathrm{sec}$ after the impact of the electron pulse there is a significant effect of $\gamma$-flush: it produces large-amplitude pile-up pulses but fortunately the pulse is not blocked by the preamplifier. This spectrometer is expected to be used in a intense neutron facility so that, in order for the preamplifier not to be dead, the center module of the EUROBALL cluster is segmented to six.
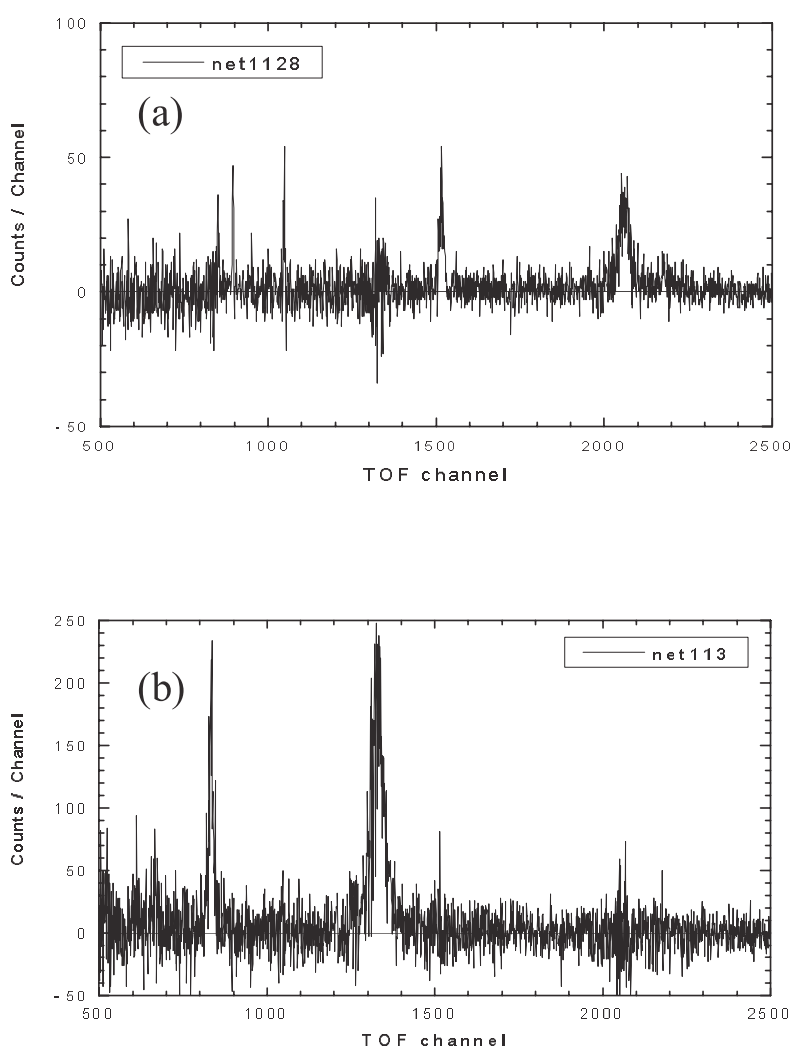

Fig. 6. TOF spectra gated for ground-state transitions of (a) $1128 \mathrm{keV}$ in ${ }^{105} \mathrm{Pd}$ and (b) $113 \mathrm{keV}$ in ${ }^{108} \mathrm{Pd}$.

In order to derive neutron capture cross section based on high-resolution Ge detectors there are mainly two methods: one utilizes ground-state transition and the other primary transition, for the identification of any cascade. Thus, prior to the TOF experiment, we need to know the decay scheme of the capture state. Generally the $\gamma$-ray intensity of primary transition is more fragmented than those of ground-state transitions. Thus we adopt the ground-state transition method in the following analysis.

\subsection{TOF spectrum}

To demonstrate the nuclide discrimination, we used a natural $\mathrm{Pd}$ sample, containing ${ }^{105} \mathrm{Pd}$ and ${ }^{108} \mathrm{Pd}$ with $22.3 \%$ and $26.5 \%$ abundances, respectively. The sample size was $18 \times 18 \times$ $1 \mathrm{~mm}$. Figure 4 shows the TOF spectrum obtained by measuring the sample for 6.6 hours. The channel division is $100 \mathrm{~ns}$ per channel. In the spectrum we can see clearly resonances at $38.4,30.2,25.2,13.2$, and $11.8 \mathrm{eV}$ from ${ }^{105} \mathrm{Pd}$, and at $33.1 \mathrm{eV}$ from ${ }^{108} \mathrm{Pd}$.

\subsection{Gamma-ray spectrum gated on TOF spectrum}

Figure 5 shows the $\gamma$-ray spectrum with G1, G2 and G3 gates on the TOF spectrum shown in figure 4 . There are several transitions originating from ${ }^{105} \mathrm{Pd}$ and the peaks at 512 
and $1128 \mathrm{keV}$ correspond to ground-state transitions. On the other hand, many peaks of ${ }^{108} \mathrm{Pd}$ are observed in the G2 spectrum and we could assign eight transitions of 113, 245, $266,276,291,325,340$, and $430 \mathrm{keV}$ as ground-state transitions.

\subsection{Gated TOF spectrum and nuclide discrimination}

We put gates on the $\gamma$-ray peaks in figure 4 and derive TOF spectra as shown in figure 6 (a) and (b). The statistics are not sufficient but it is clear in figure 6 (a) that the resonance at $33.1 \mathrm{eV}$, which is known to belong to ${ }^{108} \mathrm{Pd}$, disappears and we can observe clearly the resonances at 25.2 and $13.2 \mathrm{eV}$ of ${ }^{105} \mathrm{Pd}$. On the contrary, in figure 6 (b) we can select the resonance of $33.1 \mathrm{eV}$ from ${ }^{108} \mathrm{Pd}$. Thus it is demonstrated that by measuring single $\gamma$-rays from the sample we can distinguish nuclides which are contained in the sample itself, beam ducts, detector shield material and so on; and we can get clean TOF spectrum for a single nuclide. The experiments for ${ }^{237} \mathrm{~Np},{ }^{241} \mathrm{Am}$ and ${ }^{243} \mathrm{Am}$ are under way and a part of the results is shown in ref. [12].

\subsection{Future prospects}

The $4 \pi$ Ge spectrometer will sit in KURRI until FY2008. In 2008 it will be moved to Materials and Life Science Facility (MLF) at JAEA Proton Accelerator Research Complex (J-PARC) [13]. In MLF we can utilize $3 \mathrm{GeV}$ proton beam with a $0.33 \mathrm{~mA}$ beam intensity, producing $1 \mathrm{MW}$ power. The beam pulse width is $0.7 \mu \mathrm{sec}$ and $0.1 \mu \mathrm{sec}$ for double and single bunch mode, respectively. The pulse repetition rate is $25 \mathrm{~Hz}$. At $21.5 \mathrm{~m}$ apart from the neutron source, we will place the $4 \pi \mathrm{Ge}$ spectrometer. We are now constructing a new beam line for the TOF experiment and it will complete in FY2007. We will get the first beam in FY2008 and start the experiment in FY2009.

\section{Summary}

We have constructed a $4 \pi$ Ge spectrometer for neutron capture cross section measurement of minor actinides and longlived fission products. The spectrometer comprises two EUROBALL cluster and four clover detectors. We can measure prompt $\gamma$-rays from the sample with high accuracy. The high resolution peaks in one dimensional prompt $\gamma$-ray spectra cam be used to distinguish between individual nucleus and other sources of backgrounds. By using nat $\mathrm{Pd}$ sample it is demonstrated that contribution from several backgrounds can be removed efficiently, insuring accurate determination of neutron capture cross sections. The spectrometer will be moved to a high-intensity pulsed neutron beam facility J-PARC in FY2008 and TOF experiments will start in FY2009.

This work was supported by the Ministry of Education, Culture, Sports, Science and Technology in Japan under the project "Fundamental R\&D on Neutron Cross Sections for Innovative Reactors using Advanced Radiation Measurement Technology".

\section{References}

1. M. Igashira et al., AIP Conf. Proc. 769, 601 (2005).

2. GAMMASPHERE proposal, No. LBL-PUB-5202, 1988.

3. I.Y. Lee, Nucl. Phys. A 520, 641c (1990) .

4. J. Gerr, R.M. Lieder (Eds.), EUROBALL, A European $\gamma$-ray facility, GSI, 1992.

5. M. Sugawara et al., J. Nucl. Sci. Technol. 39, 1229 (2004).

6. J. Goto et al., AIP Conference Proc. 769, 788 (2005).

7. A. Kimura et al., AIP Conference Proc. 769, 792 (2005).

8. A. Kimura et al. (these proceedings).

9. S. Agostinelli, J. Allison, K. Amako, J. Apostolakis, H. Araujo, P. Arce, M. Asai, D. Axen, S. Banerjee, G. Barrand et al., Nucl. Instrum. Meth. A 506, 250 (2003). Geant collaboration.

10. K. Wisshak et al., Nucl. Instrum. Meth. A 292, 595 (1990).

11. CERN Report CERN/INTC-O-011 INTC-2002-037.

12. M. Mizumoto et al. (these proceedings).

13. http://j-parc.jp/index-e.html 